\title{
INDICADORES PARA LA EVALUACIÓN DE REPOSITORIOS INSTITUCIONALES DE ACCESO ABIERTO
}

\author{
Rocío Serrano Vicente* \\ Servicio de Bibliotecas. Universidad de Navarra. \\ Remedios Melero Melero** \\ Instituto de Agroquímica y Tecnología de Alimentos-CSIC. \\ Ernest Abadal*** \\ Facultad de Biblioteconomía y Documentación. Universidad de Barcelona.
}

\begin{abstract}
Resumen: Se ha realizado un estudio para definir indicadores de evaluación de repositorios, enfocados a la gestión interna y a la satisfacción de los usuarios. No existe una sola forma de evaluar los repositorios, aunque sí se pueden aplicar unos criterios generales. Se ha realizado una recopilación de trabajos sobre criterios aplicados a la evaluación y análisis de repositorios institucionales y a partir de éstos, se ha establecido una propuesta propia aplicable a repositorios institucionales como aproximación a su potencial de evaluación. Se tendrán en cuenta en esta propuesta los factores que influyen en el rendimiento de los repositorios institucionales referentes a la gestión del repositorio. Como resultado, se proponen una serie de indicadores tecnológicos, de procesos, de contenidos, de marketing y de personal, que colaboren a que el repositorio sea una herramienta útil al usuario final y a la institución.
\end{abstract}

Palabras clave: indicadores; evaluación; repositorios institucionales; acceso abierto.

Title: INDICATORS FOR THE EVALUATION OF OPEN ACCESS INSTITUTIONAL REPOSITORIES.

Abstract: After ten years from Berlin's declaration, it could be the adequate time to define some repositories evaluation indicators, focus on internal management and users' satisfaction. The approach is that even there is not an only way to evaluate institutional repositories, some general criteria could be given. After making a review of the recent literature about repositories indicators, it has been developed a set of criteria that could be applied to the institutional repositories as an evaluation potential model. It has been had in mind specifically those factors about the institutional repositories' performance related to its management. As a result, a proposal of technological, process, marketing and staff indicators is presented, the final objective being that the repository could be a useful tool for the final user and the institution.

Keywords: indicators; assessment; institutional repositories; open access.

\section{INTRODUCCIÓN.}

El acceso abierto a la ciencia significa la eliminación tanto de barreras económicas como de aquellas derivadas de los derechos de explotación que limitan la difusión, el acceso y la reutilización de recursos resultado de la producción científica y académica. Las dos vías propuestas para el acceso abierto son la ruta dorada o la publicación en revistas de acceso abierto, y la ruta verde que implica el depósito de esta producción en repositorios institucionales o temáticos de acceso abierto. La creación de los repositorios institucionales procede en buena parte del apoyo de las universidades a la iniciativa del acceso abierto y sirven para albergar en una misma plataforma la documentación digital (académica, docente, institucional, etc.) que produce la universidad. El crecimiento de los repositorios institucionales ha sido relevante sobre todo después de la Declaración de Berlín (2003) sobre acceso abierto al conocimiento, cuyos principios sobre los que descansa el acceso abierto siguen vigentes como se manifestó en el documento elaborado hace dos años en conmemoración del décimo aniversario de la Declaración de Budapest (Budapest Open Access Initiative, 2012).

Después de estos doce años, puede ser un buen momento para evaluar la calidad de los repositorios institucionales en sí y de los servicios que ofrecen al usuario, es decir, de analizar cómo los contenidos y los servicios creados a partir de éstos satisfacen las necesidades de los investigadores, principales colaboradores y destinatarios de los repositorios. La elección de unos indicadores de evaluación ayudará a examinar los repositorios y a concretar si realmente cumplen

\footnotetext{
*rserranov@unav.es

**rmelero@iata.csic.es

***abadal@ub.edu
}

Recibido: 07-02-2014; 1ª versión: 02-06-2014; 2ª versión: 20-07-2014; aceptado: 18-09-2014.

SERRANO VICENTE, R.; MELERO MELERO, R. y ABADAL, E. Indicadores para la evaluación de repositorios institucionales de acceso abierto. Anales de Documentación, 2014, vol. 17, $\mathrm{n}^{\circ} 2$ 2. Disponible en DOI: http://dx.doi.org/10.6018/analesdoc.17.2.190821 
con los objetivos para los que fueron creados. Estos indicadores pueden dar también idea de cómo han evolucionado los repositorios y de qué manera han contribuido al avance del acceso abierto a la investigación.

Parece claro que la evaluación de los repositorios depende en gran medida de sus objetivos, y de la intención que tenga la institución que lo aloja (Thibodeau, 2007). Este autor propone varios indicadores, tales como los servicios (servicios de valor añadido), la orientación y el alcance. Thibodeau se refiere a alcance en tres casos: si cumple los objetivos principales (repositorio retrospectivo, de investigación, temático), el grado de colaboración con otros repositorios (es decir, si cada repositorio cumple con todas las funciones, o estas están distribuidas entre un conjunto de depósitos digitales) y, por último, el estado de desarrollo. Es distinto evaluar un repositorio en sus inicios, que cuando ha madurado, o cuando se está realizando un cambio de estructura. Por lo tanto, no puede haber una sola forma de evaluar los repositorios, aunque sí se pueden dar unos criterios generales que puedan ayudar a los gestores de repositorios a conocer hacia dónde se puede avanzar en este campo.

En un informe publicado por SPARC (The Scholarly Publishing \& Academic Resources Coalition), los repositorios institucionales se definen como académicos, acumulativos y permanentes, abiertos e interoperables (Crow, 2002). Este autor indica también el impacto que tienen los repositorios sobre las bibliotecas, los investigadores y profesores, los editores y las agencias gubernamentales u otras entidades financiadoras de la investigación. Para las bibliotecas la creación de un repositorio institucional transforma su función de un simple guardián de la información, a un agente activo en la comunicación académica. Este hecho, además, aumenta la visibilidad de la biblioteca dentro de la propia institución. En cuanto a los profesores e investigadores, en este informe se describen claramente las dificultades existentes para que se adapten a este nuevo modelo: problemas con cuestiones relacionadas con el copyright, resistencia al cambio, etc. Pero también se mencionan las ventajas que les reporta: más visibilidad, mayor impacto de la publicación, más facilidad para defender la titularidad de las obras, etc. Los materiales docentes presentes en el repositorio se convierten en un apoyo para las clases. Este informe indica también las dificultades a las que se enfrentan las editoriales comerciales, para las que el acceso abierto supone cambiar el modelo de negocio. Solo aquellas que sepan modificar sus políticas y adaptarse a la nueva situación podrán sobrevivir a este cambio. Por último, también se prevé que las organizaciones que financian la investigación puedan adherirse al acceso abierto e impongan algún tipo de mandato para dar más rendimiento a los resultados de investigación (Crow, 2002). Por tanto, Crow se anticipaba a lo que ha sido el desarrollo del acceso abierto y los repositorios institucionales en los últimos años.

Del mismo modo que con la aparición de las bibliotecas digitales se buscaron sistemas para su evaluación, también se han propuesto algunas alternativas para evaluar la calidad de los repositorios, basadas principalmente en métodos de auditoría, con los que cada institución pueda comprobar si su repositorio cumple o no con un grupo de indicadores que para cada institución sea de interés. Estos indicadores pueden ser de distintos tipos, dirigiéndose más hacia aspectos tecnológicos (Barrueco Cruz et al., 2010), al marketing (Vierkant, 2013), o al enfoque del repositorio como un servicio dentro de la universidad (Thibodeau, 2007). Se trata de observar el grado de cumplimiento de unos indicadores, para que se puedan plantear unos objetivos de mejora en el futuro. Este será también nuestro propósito.

\section{OBJETIVOS Y METODOLOGÍA.}

El objetivo de este trabajo es la recopilación de las fuentes bibliográficas publicadas durante los años 2003-2013 que tratan sobre criterios aplicados a la evaluación y el análisis de repositorios institucionales y a partir de éstos, establecer una propuesta propia que pueda ser aplicada a los repositorios institucionales como modelo potencial de evaluación.

Se tendrán en cuenta en esta propuesta los factores que influyen en el mejor rendimiento de los repositorios institucionales referentes a la gestión del repositorio. Interesa conocer si están bien definidos los flujos de depósito y retirada de contenidos, los permisos de los usuarios y qué tipo de material se permite depositar. Además, se incluyen indicadores relacionados con el marketing interno y externo del repositorio, a través de internet, redes sociales, campañas de promoción, material promocional, etc. Parece también importante concretar indicadores referentes al personal dedicado al repositorio institucional o que colaboran con el mismo.

Por lo tanto, se pretende definir unos indicadores, que ayuden a los repositorios a evaluarse según ciertos criterios. En una primera etapa de este estudio, se persigue definir unos indicadores y la forma de medirlos en los repositorios de universidades españolas. En próximos trabajos, se expondrán los resultados de la aplicación de estos indicadores. 
La metodología utilizada ha sido la revisión bibliográfica. Se han recopilado las propuestas de indicadores publicadas durante los últimos diez años y, a partir de aquí y del análisis de la evolución del objeto de estudio, se presenta una propuesta propia. Los indicadores se agrupan en cinco grandes categorías y para cada una de ellas se ofrece una breve descripción así como la fuente de obtención de datos.

\section{LA EVALUACIÓN DE REPOSITORIOS.}

Al principio, los estudios sobre evaluación de repositorios se orientaron más bien a analizar cómo se podía aumentar el número de objetos digitales albergados, más adelante los trabajos sobre evaluación se dirigieron también a estudiar otros elementos, como los tecnológicos, los procesos, el personal, los económicos, etc. Este control perseguía, sobre todo, alinear los objetivos del repositorio con los de la institución (Cassella, 2010). Durante estos últimos años, se ha producido un permanente desarrollo tecnológico y un incremento de las aplicaciones en los repositorios. Asimismo, el crecimiento del número de instituciones que requieren el depósito de la producción científico-académica de su personal, hace que la evaluación se vaya orientando hacia otros aspectos, como por ejemplo la medición del grado de cumplimiento de las políticas institucionales de acceso abierto.

En uno de los primeros estudios de evaluación, Westell (2006) analizó la presencia de nueve indicadores en los repositorios de un grupo de universidades canadienses de habla inglesa mediante una encuesta en la que se preguntaba:

- si existía un mandato institucional (políticas)

- cómo era la integración con la planificación de la institución (apoyo institucional)

- qué modelo de financiación tenían (factores económicos)

- cuál era la relación con los centros de digitalización (retrospectivo)

- si tenían algún método de medición (tener una masa de documentos que dé estabilidad al repositorio)

- qué grado de interoperabilidad tenían (conectividad con otros repositorios)

- cómo se hacía la promoción (marketing)

- si existía una estrategia de preservación

Alma Swan, refiriéndose a la estrategia de los repositorios digitales, consideraba importantes cuatro elementos: los contenidos, el conocimiento de los usuarios, el flujo de trabajo del repositorio y la financiación (Swan, Houghton, 2012). En cuanto a los contenidos, se podría estudiar el porcentaje anual de resultados de investigación actual introducidos en el repositorio de diferentes tipos (artículos de revistas, actas de congresos, tesis); porcentaje de investigación de años anteriores recuperados y depositados; colecciones especiales digitalizadas y almacenadas. Otro indicador a estudiar es el conocimiento y la implicación de los autores, que pueden medirse en el nivel de conocimiento de los autores del acceso abierto, y de los problemas de derechos de autor, así como del nivel de conocimiento de los problemas y desarrollos de la comunicación académica. En cuanto a las prácticas del flujo de trabajo, sería interesante examinar cinco puntos: si los procedimientos tienen la suficiente calidad, si los tiempos de rendimiento son estables o mejoran, si se anticipan y suavizan los picos y los tiempos de menos trabajo, o si el repositorio está inmerso en la institución. La financiación es fundamental para poder avanzar en los aspectos tecnológicos.

La categorización de los indicadores resulta interesante porque permite sistematizar su análisis. En este sentido, el estudio llevado a cabo con repositorios institucionales coreanos se dividieron los indicadores en cuatro categorías (Kim, Kim, 2008) y cada una de ellas contenía hasta seis posibles indicadores como se muestra en la Tabla I.

\begin{tabular}{|l|l|l|l|l|l|l|}
\hline Categorías & Indicador 1 & Indicador 2 & Indicador 3 & Indicador 4 & Indicador 5 & Indicador 6 \\
\hline Contenido & Metadatos & Tamaño & $\begin{array}{l}\text { Características } \\
\text { del } \\
\text { procedimiento }\end{array}$ & $\begin{array}{l}\text { Características } \\
\text { formales }\end{array}$ & & \\
\hline $\begin{array}{l}\text { Gestión y } \\
\text { política }\end{array}$ & $\begin{array}{l}\text { Criterios de } \\
\text { procedimiento } \\
\text { (legales y } \\
\text { costes) }\end{array}$ & $\begin{array}{l}\text { Apoyo de la } \\
\text { institución }\end{array}$ & $\begin{array}{l}\text { Participación } \\
\text { de profesores }\end{array}$ & & & \\
\hline Sistema & Acceso & Integración & $\begin{array}{l}\text { Confianza en } \\
\text { el sistema }\end{array}$ & Interoperabilidad & Funcionalidad & Cooperación \\
\hline $\begin{array}{l}\text { Uso } \\
\text { usuarios }\end{array}$ & Accesibilidad & Usabilidad & $\begin{array}{l}\text { Satisfacción } \\
\text { de usuarios }\end{array}$ & Relevancia & $\begin{array}{l}\text { Cumplimiento } \\
\text { de plazos }\end{array}$ & $\begin{array}{l}\text { Usuarios } \\
\text { potenciales }\end{array}$ \\
\hline
\end{tabular}

Tabla I. Indicadores estudiados en los repositorios institucionales de Corea (Kim, Kim, 2008). 
Este estudio se realizó mediante entrevistas a bibliotecarios, gestores de biblioteca e investigadores siguiendo el método Delphi y sesiones de focus group. Los resultados indicaron que no coincidían los indicadores que los bibliotecarios consideraban más importantes (como los metadatos o la aceptación de los gestores), con los que realmente parecían que estaban más relacionados con el desarrollo de los repositorios (como la tasa de uso y los recursos económicos). Es un estudio teórico quizá superado ya por el acelerado avance de la tecnología, pero es un buen resumen de la variedad de indicadores a tener en cuenta.

Maria Cassella (Cassella, 2010) dispone de un estudio más reciente de indicadores de evaluación del rendimiento de repositorios, que puede ser de gran utilidad. Estos indicadores los divide en internos, que pueden ayudar a los gestores de repositorios a alinear sus estrategias con la misión y objetivos institucionales, priorizando aquellos indicadores que coincidan más con éstos; y en externos que pueden ayudar a los gestores a medir el impacto del repositorio a nivel nacional e internacional y tasar su valor como una herramienta de búsqueda para usuarios finales (Tabla II).

\begin{tabular}{|l|l|l|}
\hline Internos & $\begin{array}{l}\text { Perspectiva del } \\
\text { usuario/depositante }\end{array}$ & Contenido, quien deposita, ratios \\
\hline & Procesos internos & $\begin{array}{l}\text { Depósitos anuales, depósitos diarios, acceso a } \\
\text { texto completo, colecciones }\end{array}$ \\
\hline & Servicios de valor añadido & $\begin{array}{l}\text { Cvs, estadísticas, IDs autor, guías, FAQs, } \\
\text { vocabularios controlados, add-ons, alertas RSS, } \\
\text { atom feeds }\end{array}$ \\
\hline Externos & $\begin{array}{l}\text { Anteroperabilidad desarrollo del } \\
\text { personal }\end{array}$ & $\begin{array}{l}\text { Personal dedicado, personas implicadas, } \\
\text { formación del personal }\end{array}$ \\
\hline & $\begin{array}{l}\text { Financiación externa } \\
\text { Participación en proyectos } \\
\text { nacionales e internacionales }\end{array}$ & $\begin{array}{l}\text { Interacción con otras redes de repositorios } \\
\text { consorciados (para unir esfuerzos) } \\
\text { indicador de la visibilidad y la reputación }\end{array}$ \\
\hline
\end{tabular}

Tabla II: Indicadores para la evaluación de repositorios institucionales propuestos por Cassella (2010).

En diciembre de 2010, se publicó una guía editada por REBIUN, FECYT y RECOLECTA (Barrueco Cruz et al., 2010) donde se recogían, en siete secciones, 31 criterios de evaluación que están basados en proyectos ya existentes en Europa (DRIVER y DINI). Es una relación de criterios muy detallada y fácil de comprobar. Las secciones en que está dividida son las siguientes:

- Visibilidad: Se estudia si el repositorio está visible en la propia institución y presente en los directorios nacionales e internacionales. También se refiere a la proporción de documentos del repositorio en acceso abierto.

- Políticas: Los indicadores de este apartado se refieren a si existe una definición de la misión y objetivos del repositorio, y si existe una política pública sobre depósito de documentos, preservación de contenidos y reutilización de metadatos.

- Aspectos legales: Se comprueba si existe una autorización del autor de cada documento introducido en el caso de que el depósito sea mediado, y si está disponible información sobre derechos de autor en el repositorio.

- Metadatos: Estos indicadores van dirigidos a conocer si los propios metadatos siguen una política de indización, y si se permite la exportación de metadatos en algún otro formato que el Dublin Core.

Aunque esta guía se realizó en diciembre de 2010, no ha sido posible hasta ahora realizar la encuesta entre los repositorios españoles. Se acaba de publicar la nueva versión de esta guía redactada por un nuevo grupo de trabajo de REBIUN y FECYT para llevar a cabo esta evaluación de los repositorios de investigación (Barrueco-Cruz, et al., 2014).

Por otro lado, la encuesta que realizó el Primary Research Group (2012) sobre repositorios institucionales, en la que participaron universidades de todo el mundo, definió un grupo de indicadores que podrían servir de guía para 
estudiar la calidad de un grupo de repositorios nacionales. Se trata de una lista exhaustiva de indicadores, entre los que se pueden destacar los que se muestran en la Tabla III.

\begin{tabular}{|c|c|}
\hline Marketing & $\begin{array}{l}\text { Enlace a la biblioteca y a la página de inicio de la institución } \\
\text { Enlaces al canal de Youtube de la universidad } \\
\text { Enlaces al sitio iTunes de la universidad } \\
\text { Blog propio } \\
\text { Folleto informativo } \\
\text { Podcast y webcast regulares } \\
\text { Publicación del informe anual } \\
\text { Notas de prensa } \\
\text { Presentaciones en eventos } \\
\text { Enlaces externos y posicionamiento en motores de búsqueda } \\
\text { Presupuesto dedicado al marketing }\end{array}$ \\
\hline Acceso y estadísticas & $\begin{array}{l}\text { Número de visitantes por año } \\
\text { Número de descargas anuales } \\
\text { Procedencia de las descargas (externas e internas) } \\
\text { Informes periódicos a los autores sobre descargas } \\
\text { Interfaz de los autores para gestionar las descargas recibidas } \\
\text { Servicio autor-usuario para resumir las descargas o visitas recibidas, p. ej., por email } \\
\text { Uso del repositorio (visitas, descargas, páginas vistas) } \\
\text { Definición de las estadísticas } \\
\text { Promoción }\end{array}$ \\
\hline Asuntos económicos & $\begin{array}{l}\text { Personal dedicado al repositorio, tipo y procedencia (biblioteca, departamentos, } \\
\text { servicios técnicos) } \\
\text { Presupuesto }\end{array}$ \\
\hline Cooperación & $\begin{array}{l}\text { Tanto por ciento de depósito de la producción institucional } \\
\text { Campaña de difusión entre el personal facultativo } \\
\text { Promoción y campañas de las ventajas de la participación en el depósito }\end{array}$ \\
\hline Impacto & $\begin{array}{l}\text { Control de citas } \\
\text { Impacto en general del repositorio }\end{array}$ \\
\hline $\begin{array}{l}\text { Catalogación, gestión de } \\
\text { derechos }\end{array}$ & $\begin{array}{l}\text { Tipos de documentos } \\
\text { Acceso al texto completo: antes de la publicación, cuando se publica, o con un } \\
\text { embargo después de la publicación } \\
\text { Catalogación por la biblioteca }\end{array}$ \\
\hline Financiación del repositorio & $\begin{array}{l}\text { Ayudas específicas } \\
\text { Del presupuesto de la biblioteca } \\
\text { De los dos anteriores }\end{array}$ \\
\hline Contenido & Tipos de documentos que contiene el repositorio \\
\hline
\end{tabular}

También cabe destacar un censo realizado de los repositorios institucionales alemanes (Vierkant, 2013), donde se estudiaron los indicadores siguientes:

- Tamaño

- Dónde se alojan

- Idiomas a los que está traducido

- Servicios de valor añadido: posibilidad de exportar las citas, estadísticas de uso, RSS, marcadores sociales a nivel de ítem, posibilidad de enlazar con redes sociales a nivel de ítem

- Software

- Formato de metadatos: Dublin Core simple, Dublin Core cualificado, XMetaDiss, XMetaDissPlus

- Registros en páginas de repositorios

- Firma de declaraciones de acceso abierto

De acuerdo con los trabajos previos se ha comprobado que las categorías estudiadas dependen del enfoque desde el que se lleva a cabo la evaluación, que suele ser distinto en cada caso. De los ocho casos estudiados, algunas categorías que se repiten en algunos de ellos son el marketing (Westell, 2006; Cassella, 2010; Primary Research Group, 2012; Vierkant, 2013), los factores económicos y la financiación (Westell, 2006; Swan, Houghton, 2012; Cassella, 2010; 
Primary Research Group, 2012), la interoperabilidad y cooperación (Westell, 2006, Thibodeau, 2007; Kim, Kim, 2008; Cassella, 2010; Vierkant, 2013), las políticas del repositorio (Westell, 2006; Kim, Kim, 2008; Barrueco Cruz et al., 2010; Vierkant, 2013), los contenidos (Swan, Houghton, 2012), y los servicios de valor añadido (que dependen en gran medida del personal que trabaje en este ámbito) (Thibodeau, 2007; Cassella, 2010; Vierkant, 2013). En la Tabla IV se comparan estos casos por categorías/autor en cuanto a los indicadores relacionados con estas categorías. En el estudio realizado se comprueba que los enfoques de evaluación de repositorios son muy variados, de acuerdo con el punto de vista que se estudie. A partir de la bibliografía consultada, y de la experiencia que se ha recogido tanto en la gestión de repositorios, como en la colaboración en una de las herramientas de indicadores, se deduce que se puede hacer una propuesta de indicadores que evalúen los repositorios, no de un modo absoluto, sino desde varias perspectivas. 


\begin{tabular}{|c|c|c|c|c|c|c|c|c|}
\hline Categorías/Autores & Westell (2006) & Thibodeau (2007) & Swan (2012) & Kim (2008) & Cassella (2010) & PRG (2012) & FECYT (2010) & Vierkant (2013) \\
\hline Marketing & Medición & & & & Estadísticas & $\begin{array}{l}\mathrm{N}^{\circ} \text { visitantes por año } \\
\mathrm{N}^{\circ} \text { descargas....(ver tabla } \\
\mathrm{IV} \text { ) }\end{array}$ & & $\begin{array}{l}\text { Registros en pági- } \\
\text { nas de repositorios } \\
\text { Redes sociales }\end{array}$ \\
\hline Económicos & $\begin{array}{l}\text { Modelo de financia- } \\
\text { ción }\end{array}$ & & $\begin{array}{l}\text { Planes de financiación } \\
\text { anuales, monitorizar } \\
\text { procedimientos, prever } \\
\text { procesos }\end{array}$ & & $\begin{array}{l}\text { Capacidad del } \\
\text { repositorio de atraer } \\
\text { financiación }\end{array}$ & $\begin{array}{l}\text { Presupuesto, ayudas es- } \\
\text { pecíficas }\end{array}$ & & \\
\hline $\begin{array}{l}\text { Interoperabilidad } \\
\text { cooperación }\end{array}$ & $\begin{array}{l}\text { Conectividad con } \\
\text { otros repositorios }\end{array}$ & $\begin{array}{l}\text { Reparto de tareas con } \\
\text { otros repositorios }\end{array}$ & & Interoperabilidad & $\begin{array}{lr}\text { Interacción } & \text { con } \\
\text { otras redes de } \\
\text { repositorios con- } \\
\text { sorciados (para } \\
\text { unir esfuerzos) }\end{array}$ & & & $\begin{array}{l}\text { Idiomas en los } \\
\text { que se puede } \\
\text { buscar } \\
\text { Formato de me- } \\
\text { tadatos }\end{array}$ \\
\hline Políticas & Mandato & & & $\begin{array}{l}\text { Apoyo de la } \\
\text { institución }\end{array}$ & & & $\begin{array}{l}\text { Política pública sobre el } \\
\text { depósito de documentos }\end{array}$ & $\begin{array}{l}\text { Firmas de declara- } \\
\text { ciones de acceso } \\
\text { abierto }\end{array}$ \\
\hline Contenidos & & & $\begin{array}{l}\text { Porcentaje anual de } \\
\text { resultados de investiga- } \\
\text { ción actual introducidos } \\
\text { en el repositorio de } \\
\text { diferentes tipos Porcen- } \\
\text { taje de investigación de } \\
\text { años anteriores recupe- } \\
\text { rados y depositados }\end{array}$ & & & & & \\
\hline $\begin{array}{l}\begin{array}{l}\text { Servicios } \\
\text { añadido }\end{array} \\
\text { and }\end{array}$ & & $\begin{array}{lll}\text { Servicios } & \text { de } & \text { valor } \\
\text { añadido } & & \\
\end{array}$ & & & $\begin{array}{l}\text { Personal dedicado, } \\
\text { personas implicadas, } \\
\text { formación del } \\
\text { personal }\end{array}$ & & & $\begin{array}{l}\text { Posibilidad de } \\
\text { exportar las citas, } \\
\text { estadísticas de uso, } \\
\text { RSS, Marcadores } \\
\text { sociales a nivel de } \\
\text { ítem, posibilidad de } \\
\text { enlazar con redes } \\
\text { sociales por ítem. }\end{array}$ \\
\hline
\end{tabular}

Tabla IV: Comparación de los indicadores propuestos en la bibliografía revisada. 
Este trabajo aunque estudia también los aspectos tecnológicos, se centra especialmente en los aspectos relacionados con la gestión del repositorio, los servicios de valor añadido y el personal. Es decir, se pretende ver cómo la organización interna puede influir en el mayor o menor éxito de un repositorio. El mejor método para aplicar estos indicadores parece una encuesta a los gestores de los repositorios, así como la revisión de los repositorios universitarios españoles, aunque no se descartan otras fórmulas que incluyan un análisis cualitativo a través de entrevistas personales.

\section{PROPUESTA DE INDICADORES.}

\subsection{Cuestiones preliminares.}

El repositorio institucional en el marco de las universidades se ha definido como un conjunto de servicios que ofrece la universidad a los miembros de su comunidad para la gestión y difusión de los materiales digitales creados por la propia institución y sus componentes (Lynch, 2003). En este sentido son importantes varios puntos a estudiar relacionados con la gestión del repositorio: los procedimientos de depósito de documentos, con especial atención a la interoperabilidad con otros sistemas (CRIS, OPAC, etc.), los datos de estadísticas que se proporcionan y cómo se hacen llegar al usuario, y el marketing interno del producto.

La biblioteca puede considerar, o no, el repositorio institucional como un punto clave en su servicio. Por eso, se podría estudiar qué recursos económicos, y, sobre todo, de personal se dedica a su gestión y mantenimiento. Y la formación y categoría del personal encargado de estas tareas.

En cuanto al usuario externo, se podría estudiar si el repositorio está conectado con motores de búsqueda y en concreto con cuáles. También sería interesante conocer si conectan con sistemas de referencias bibliográficas; si el programa puede hacer búsquedas de calidad dentro del repositorio, y si los canales de preguntas y respuestas son claros y se gestionan con agilidad. Estos aspectos no solo son aplicables a los usuarios de otras instituciones, sino a los propios usuarios, que utilizan la información disponible en otros repositorios españoles.

En conclusión, esta evaluación de repositorios tiene dos aspectos:

- Factores técnicos o internos al repositorio, como por ejemplo la tecnología, los procesos, la gestión, o la interoperabilidad con otros repositorios

- Factores institucionales: cómo se integra el repositorio con las políticas de la institución, adaptándose a las necesidades de la universidad y de los investigadores. Es decir, cómo se alinea con esos objetivos de la institución, y cómo son los servicios a los investigadores

El éxito del repositorio viene definido fundamentalmente por estos últimos aspectos. Si realmente está integrado y los investigadores son colaboradores del mismo, el repositorio será la imagen de la investigación de la institución.

\subsection{Indicadores propuestos.}

Se han establecido un total de 32 indicadores, agrupados en cinco categorías.

\section{Tecnología}

\begin{tabular}{|c|c|c|}
\hline Indicador & Definición & Obtención de datos \\
\hline Tipo de software & Tipo de software utilizado & Encuesta a bibliotecarios \\
\hline Diseño & $\begin{array}{l}\text { La interfaz sigue la identidad gráfica de la } \\
\text { universidad }\end{array}$ & Consulta de la web \\
\hline Preservación & $\begin{array}{l}\text { Conocer si tienen política de preservación } \\
\text { de datos }\end{array}$ & Consulta de la web \\
\hline Web 2.0 & $\begin{array}{l}\text { Relación con redes sociales en cada } \\
\text { documento }\end{array}$ & Consulta al repositorio \\
\hline $\begin{array}{l}\text { Tipo de autentificación de } \\
\text { los usuarios }\end{array}$ & P. ej.: LDAP, usuario/password & $\begin{array}{l}\text { Encuesta a los gestores de } \\
\text { repositorios }\end{array}$ \\
\hline $\begin{array}{l}\text { Relación con sistemas } \\
\text { internos de la universidad }\end{array}$ & $\begin{array}{l}\text { Conocer si el repositorio se comunica con } \\
\text { los CRIS, servicio de publicaciones }\end{array}$ & $\begin{array}{l}\text { Encuesta a los gestores de } \\
\text { repositorios }\end{array}$ \\
\hline Estadísticas de uso & Tipo de estadísticas que se utilizan & $\begin{array}{l}\text { Comprobar en repositorio / } \\
\text { Encuesta a los gestores }\end{array}$ \\
\hline
\end{tabular}




\section{Procedimientos}

\begin{tabular}{|c|c|c|}
\hline Indicador & Definición & Obtención de datos \\
\hline $\begin{array}{l}\text { Retirada de objetos } \\
\text { digitales }\end{array}$ & $\begin{array}{l}\text { Conocer si existe una política de retirada de } \\
\text { materiales (p. ej.: en caso de fraude, libelo o } \\
\text { incumplimiento de contratos) }\end{array}$ & $\begin{array}{l}\text { Encuesta a los gestores de } \\
\text { repositorios y consulta a la web }\end{array}$ \\
\hline $\begin{array}{l}\text { Existencia de } \\
\text { manuales de } \\
\text { procedimiento } \\
\end{array}$ & & $\begin{array}{l}\text { Encuesta a los gestores de } \\
\text { repositorios y consulta a la web }\end{array}$ \\
\hline $\begin{array}{l}\text { Existencia de } \\
\text { manuales de estilo }\end{array}$ & & $\begin{array}{l}\text { Encuesta a los gestores de } \\
\text { repositorios y consulta a la web }\end{array}$ \\
\hline $\begin{array}{l}\text { Tipo de usuario que } \\
\text { puede depositar }\end{array}$ & P. ej. autor, depósito mediado & $\begin{array}{l}\text { Encuesta a los gestores de } \\
\text { repositorios y consulta a la web }\end{array}$ \\
\hline $\begin{array}{l}\text { Tipo de flujos de } \\
\text { depósito }\end{array}$ & $\begin{array}{l}\text { P. ej. por tipo de documento, por tipo de } \\
\text { usuario }\end{array}$ & $\begin{array}{l}\text { Encuesta a los gestores de } \\
\text { repositorios y consulta a la web }\end{array}$ \\
\hline Derechos de autor & $\begin{array}{l}\text { Gestión de las restricciones editoriales y } \\
\text { embargos }\end{array}$ & $\begin{array}{l}\text { Encuesta a los gestores de } \\
\text { repositorios y consulta a la web }\end{array}$ \\
\hline $\begin{array}{l}\text { Importación y } \\
\text { exportación masiva }\end{array}$ & $\begin{array}{l}\text { Dispone de mecanismos de importación y } \\
\text { exportación masiva de metadatos y objetos } \\
\text { digitales. }\end{array}$ & Encuesta a gestores \\
\hline $\begin{array}{l}\text { Verificación de } \\
\text { metadatos }\end{array}$ & $\begin{array}{l}\text { Revisión de metadatos en el proceso de } \\
\text { depósito }\end{array}$ & Encuesta a gestores \\
\hline
\end{tabular}

\section{Contenidos}

\begin{tabular}{|l|l|l|}
\hline Indicador & Definición & Obtención de datos \\
\hline $\begin{array}{l}\text { Tasa de crecimiento } \\
\text { de colecciones }\end{array}$ & Registros por año & Sitio web/Encuesta gestores \\
\hline Tipos de documentos & Tipos dentro de DC & Sitio web del repositorio \\
\hline Versiones & $\begin{array}{l}\text { Versiones que se puede depositar: pre-print, post- } \\
\text { print, versión editorial }\end{array}$ & Sitio web del repositorio \\
\hline Acceso abierto & Porcentaje de documentos a texto completo & Encuesta gestores \\
\hline $\begin{array}{l}\text { Política sobre el } \\
\text { archivo en el } \\
\text { repositorio }\end{array}$ & $\begin{array}{l}\text { Documento público en el que se establece quién } \\
\text { puede aportar contenidos, tipos de contenidos, } \\
\text { formatos de fichero, y retirada de documentos }\end{array}$ & Sitio web del repositorio \\
\hline Contenidos & Patrimonial, académico, institucional, otros & Sitio web del repositorio \\
\hline $\begin{array}{l}\text { Preservación de } \\
\text { contenidos }\end{array}$ & $\begin{array}{l}\text { Formatos de documentos en que se asegure } \\
\text { preservación }\end{array}$ & Sitio web repositorio \\
\hline
\end{tabular}

\section{Marketing}

\begin{tabular}{|l|l|l|}
\hline Indicador & Definición & Obtención de datos \\
\hline $\begin{array}{l}\text { Promoción en la propia } \\
\text { institución }\end{array}$ & $\begin{array}{l}\text { Herramientas que se utilizan para fomentar el } \\
\text { conocimiento del repositorio en la propia } \\
\text { institución: sesiones de formación, fomento del } \\
\text { acceso abierto, materiales de soporte disponibles }\end{array}$ & $\begin{array}{l}\text { Encuesta a los gestores } \\
\text { de repositorios }\end{array}$ \\
\hline Hipervínculos & $\begin{array}{l}\text { Enlace desde la página web de la institución y } \\
\text { desde la página web de la biblioteca }\end{array}$ & $\begin{array}{l}\text { Encuesta a los gestores } \\
\text { de repositorios y } \\
\text { estudio de las web } \\
\text { institucional, biblioteca } \\
\text { y del repositorio, así } \\
\text { como de los blogs y } \\
\text { tutoriales }\end{array}$ \\
\hline
\end{tabular}




\begin{tabular}{|l|l|l|}
\hline $\begin{array}{l}\text { Tipo de promoción que } \\
\text { se realiza entre agentes } \\
\text { externos }\end{array}$ & $\begin{array}{l}\text { Notas de prensa, seminarios, conferencias, folletos } \\
\text { informativos }\end{array}$ & $\begin{array}{l}\text { Encuesta a los gestores } \\
\text { de repositorios }\end{array}$ \\
\hline Blog repositorio & Existe un blog propio del repositorio & Consulta web/Encuesta \\
\hline $\begin{array}{l}\text { Publicación informe } \\
\text { anual }\end{array}$ & Se publica anualmente un informe de actividad & Encuesta a gestores \\
\hline $\begin{array}{l}\text { Mecanismos para } \\
\text { incentivar el archivo }\end{array}$ & Tipo de estrategias llevadas a cabo & $\begin{array}{l}\text { Encuesta a } \\
\text { bibliotecarios }\end{array}$ \\
\hline
\end{tabular}

\section{Personal}

\begin{tabular}{|c|c|c|}
\hline Indicador & Definición & Obtención de datos \\
\hline $\begin{array}{l}\text { Tasa de bibliotecarios } \\
\text { responsables de las tareas del } \\
\text { repositorio institucional }\end{array}$ & $\begin{array}{l}\text { Cuántos bibliotecarios se dedican al } \\
\text { desarrollo del repositorio del total del } \\
\text { personal de la biblioteca }\end{array}$ & $\begin{array}{l}\text { Encuesta a los gestores } \\
\text { de repositorios }\end{array}$ \\
\hline $\begin{array}{l}\text { Responsables de la gestión y } \\
\text { mantenimiento }\end{array}$ & $\begin{array}{l}\text { Equipo repositorio } \\
\text { Todos los bibliotecarios }\end{array}$ & $\begin{array}{l}\text { Encuesta a los gestores } \\
\text { de repositorios }\end{array}$ \\
\hline Concienciación & $\begin{array}{l}\text { Conocimiento y apoyo de la dirección de la } \\
\text { biblioteca sobre el acceso abierto y el } \\
\text { repositorio }\end{array}$ & $\begin{array}{l}\text { Encuesta a los gestores } \\
\text { de repositorios, y a los } \\
\text { directores de } \\
\text { bibliotecas }\end{array}$ \\
\hline Formación & $\begin{array}{l}\text { Tasa de asistencia de los bibliotecarios a } \\
\text { sesiones de formación sobre el acceso abierto } \\
\text { sobre el total del personal }\end{array}$ & $\begin{array}{l}\text { Encuesta a los gestores } \\
\text { de repositorios }\end{array}$ \\
\hline
\end{tabular}

\section{CONCLUSIONES.}

La evaluación de repositorios institucionales de investigación es un ámbito que se ha estudiado desde varios puntos de vista, fundamentalmente formales, es decir, referidos al software utilizado, la adaptación a los distintos estándares que han ido surgiendo durante este tiempo, y que permiten una mayor unificación de los datos recogidos en ellos. Las propuestas de indicadores para la evaluación de repositorios presentadas en los últimos años han sido numerosas e incluyen diversas perspectivas o categorías de análisis.

Sin embargo, siendo muy importantes estos elementos desde el punto de vista de la comparación de datos entre unos y otros repositorios, parece interesante abordar una evaluación que se centre más en lo que hemos llamado "factores institucionales", es decir, en analizar cómo se adecúa el repositorio a las necesidades de la institución y de los investigadores (Westell, 2006; Thibodeau, 2007) que, en definitiva, son los usuarios finales de estas plataformas. La evaluación de los elementos técnicos y formales es importante, y de hecho, se está realizando, pero se considera importante evaluar el repositorio desde el punto de vista de cómo está sirviendo a la institución de la que depende para cumplir sus fines y objetivos.

En la bibliografía revisada se han dividido en seis categorías los indicadores que se proponen: marketing (cómo se promociona el repositorio) (Westell, 2006; Cassella, 2010; Primary Research Group, 2012; Vierkant, 2013); indicadores económicos, es decir, cómo se financia tanto desde la universidad como externamente; interoperabilidad y cooperación con otros repositorios, que está relacionado con la facilidad para ser recolectado y la relación con otras instituciones (Westell, 2006; Swan, Houghton, 2012; Cassella, Morando, 2012; Primary Research Group, 2012); políticas, que indica qué grado de implicación tiene la propia institución en el depósito de documentos de investigación en el repositorio (Kim, Kim, 2006; Westell, 2006; Barrueco Cruz et al., 2010; Vierkant, 2013); contenidos, que indican numéricamente si los investigadores o docentes están realmente concienciados porque introducen sus resultados en el portal (Swan, Houghton, 2012); y, por último, servicios de valor añadido, que supone el esfuerzo de cantidad de personas, y cualificación, por una parte, y por otra los servicios que se dan a los investigadores para que les interese el repositorio como herramienta de difusión de sus investigaciones (Thibodeau, 2007; Cassella, 2010; Vierkant, 2013).

En este trabajo se ha presentado una propuesta de indicadores para la evaluación y seguimiento de repositorios que incluye diversas categorías (tecnología, procesos, contenidos, marketing, personal) y que puede servir para que el 
repositorio sea una herramienta útil y adecuada al usuario final y a los objetivos de la institución que lo ha creado. Estos indicadores se pueden aplicar a nivel individual y también a grupos de repositorios pudiéndose así comparar entre ellos. Sin embargo, no se pretende con ello realizar un ranking de repositorios, sino comprobar cómo cada uno de ellos se alinea o no con la política de la institución, y si el hacerlo favorece su desarrollo.

Las dificultades para la recogida de datos se pueden minimizar con encuestas a los gestores de repositorios, que puedan aclarar en último término los ámbitos que puedan quedar menos claros a partir del estudio de las páginas web de los repositorios, y otras páginas que contienen información sobre ellos.

En una segunda etapa de la investigación, se pretende realizar un estudio a través de encuestas y focus group (Kim, Kim, 2006), para determinar el grado de conocimiento de los usuarios finales (investigadores), y su satisfacción con el repositorio, así como la opinión de los gestores de la investigación dentro de una institución. Con estos datos, se podría conocer cómo un repositorio puede gestionarse de una forma efectiva desde el punto de vista interno, y cómo esa gestión puede influir en la satisfacción del usuario final, de tal forma que considere el repositorio institucional como el camino natural para difundir su investigación. Por otro lado, el estudio se podrá comparar con el informe de situación que se llevó a cabo en 2008 el grupo de investigación Acceso Abierto a la Ciencia (Melero et al, 2009) y permitirá conocer la evolución de los repositorios españoles en los últimos años.

\section{BIBLIOGRAFÍA.}

BERLIN DECLARATION ON OPEN ACCESS TO KNOWLEDGE IN THE SCIENCES AND HUMANITIES, 2003. <http://openaccess.mpg.de/286432/Berlin-Declaration> [Consulta: 2 de junio de 2014].

BARRUECO CRUZ, J.M. et al. Guía para la evaluación de repositorios institucionales de investigación. Madrid: FECYT, RECOLECTA $\quad y \quad$ CRUE, 2010. <http://recolecta.fecyt.es/sites/default/files/contenido/documentos/GuiaEvaluacionRecolectav1.0-1.pdf> [Consulta: 23 de junio de 2014].

BARRUECO CRUZ, J.M. et al. Guía para la evaluación de repositorios institucionales de investigación. Madrid: FECYT, RECOLECTA y CRUE, 2014. <http://recolecta.fecyt.es/guias> [Consulta: 2 de junio de 2014].

BUDAPEST OPEN ACCESS INITIATIVE, 2012. Ten years on from the Budapest Open Access Initiative: setting the default to open <http://www.budapestopenaccessinitiative.org/boai-10-recommendations> [Consulta: 23 de enero de 2014].

CASSELLA, M. y MORANDO, M. Fostering new roles for librarians: skills set for repository managers — results of a survey in Italy. LIBER Quarterly, 2012, vol. 21, $\mathrm{n}^{\mathrm{o}}$ 3/4, $\mathrm{p}$. 407-428. $<$ http://liber.library.uu.nl/index.php/lq/article/view/8033/8397> [Consulta: 2 de junio de 2014].

CASSELLA, M. Institutional repositories: an internal and external perspective on the value of IRS for researchers' communities. LIBER Quarterly, 2010, vol. 20, n 2, p. 210-225.

CROW, R. The case for institutional repositories: a SPARC position paper. ARL Bimonthly Report, $223,2002$.

KIM, H.H. y KIM, Y.H. An evaluation model for the national consortium of institutional repositories of Korean universities. Proceedings of the American Society for Information Science and Technology, 2006, vol. 43, n ${ }^{\circ} 1$, p. 119. <http://dx.doi.org/10.1002/meet.1450430176> [Consulta: 2 de junio de 2014].

KIM, Y.H. y KIM, H.H. Development and validation of evaluation indicators for a consortium of institutional repositories: a case study of collection. Journal of the American Society for Information Science \& Technology, 2008, vol. 59, no 8, p. 1282-1294. <http://dx.doi.org/10.1002/asi.20818> [Consulta: 2 de junio de 2014].

LYNCH, C. Institutional repositories: essential infrastructure for scholarship in the digital age. Portal: Libraries and the Academy, 2003, vol. 3, n 2, p. 327-336.

MELERO, R.; ABADAL, E.; ABAD, F. y RODRÍGUEZ-GAIRÍN, J.M. The situation of open access institutional repositories in Spain: 2009 report. Information research, 2009, vol. 14, $\mathrm{n}^{\circ} 4 .<$ http://informationr.net/ir/144/paper415.html> [Consulta: 2 de junio de 2014].

PRIMARY RESEARCH GROUP. The survey of institutional digital repositories, 2012-13Edition. New York, NY: Primary Research Group, 2012.

SWAN, A. y HOUGHTON, J. Going for gold? The costs and benefits of gold open access for UK research institutions: further economic modelling. Report to the UK Open Access Implementation Group. 2012 $<$ http://repository.jisc.ac.uk/610> [Consulta: 16 de julio de 2014].

THIBODEAU, K. If you build it, will it fly? Criteria for success in a digital repository. Journal of Digital Information, 2007, vol. 8, n 2, p. 7. <https://journals.tdl.org/jodi/index.php/jodi/article/view/197> [Consulta: 2 de junio de 2014]. 
VIERKANT, P. Census of open access repositories in Germany: turning perceived knowledge into sound understanding. D-Lib Magazine, 2013, vol. 19, $\mathrm{n}^{\circ} 11<$ http://dx.doi.org/10.1045/november2013-vierkant> [Consulta: 16 de julio de 2014].

WESTELL, M. Institutional repositories: proposed indicators of success. Library Hi Tech, 2006, vol. 24, nº 2, p. $211-$ 226. <http://dx.doi.org/10.1108/07378830610669583> [Consulta: 2 de junio de 2014]. 